\title{
Very Wide Range Frequency Synthesizer Architecture for Avionic SDR Applications
}

\author{
Zakaria El Alaoui Ismaili ${ }^{1,2}$, Wessam Ajib ${ }^{2}$, François Gagnon ${ }^{1}$ and Frederic Nabki ${ }^{2}$ \\ ${ }^{1}$ Electrical Engineering Department, École de technologie supérieure (ÉTS), Canada \\ ${ }^{2}$ Computer Sciences Department, Université du Québec à Montréal (UQAM), Canada \\ zakaria.el-alaoui-ismaili.1@ens.etsmtl.ca, \{ajib.wessam, nabki.frederic\}@uqam.ca, francois.gagnon@etsmtl.ca
}

\begin{abstract}
This paper presents a very wide range frequency synthesizer architecture appropriate to avionic software defined radio $(S D R)$ applications. The synthesizer generates a continuous carrier frequencies range between $187 \mathrm{MHz}$ and $12 \mathrm{GHz}$ that covers most of avionic communication applications. The covered range is distributed into twenty sub-bands by using a voltage controlled oscillator (VCO). The considered VCO is able to achieve a tuning range from $10 \mathrm{GHz}$ to $12 \mathrm{GHz}(18.2 \%)$ and from $7 \mathrm{GHz}$ to $8.5 \mathrm{GHz}(19.35 \%)$. It includes a capacitor bank, varactors, and switched inductors and is designed in $0.13 \mu \mathrm{m}$ CMOS technology. Using the advanced design system (ADS) simulation tool and SpectreRF simulator, the proposed VCO exhibits a phase noise of $-125 \mathrm{dBc} / \mathrm{Hz}$ at $12 \mathrm{GHz}$ and at a $10 \mathrm{MHz}$ offset frequency with a power consumption of $4.1 \mathrm{~mW}$. At $10 \mathrm{GHz}$, the simulated synthesizer phase noise is of $-102 \mathrm{dBc} / \mathrm{Hz}$ at a $1 \mathrm{MHz}$ frequency offset. In addition, the loop bandwidth of the phase locked loop (PLL) is $1.1 \mathrm{MHz}$ whereas the settling time is 3.64 $\mu$ s.
\end{abstract}

\section{INTRODUCTION}

The benefits, defined by higher flexibility, lower implementation area, lower cost and lower power consumption of software defined radio (SDR) technology gave rise to a promising approach for future wireless communication systems. SDR technology presents also a suitable solution for integrating and improving the implementation of avionic communication systems. Engineers and system architects need to improve this implementation by minimizing the hardware congestion in aircraft in order to reduce cost and lower energy requirements. The requirements of avionic radio systems such as Distance Measuring Equipment (DME), Mode S transponder, Traffic collision avoidance system (TCAS) and Global Positioning System (GPS) require the implementation of several standards. Moreover, other standards, such as WiMAX and wireless cellular systems, need to be implemented in order to ensure better communication services and more wireless access applications. SDR systems can be used to fully integrate navigation radio systems, communication systems, automatic position reporting system and data links into a single reconfigurable module.

However, avionic communication systems operate under different frequency bands. The allocated spectrum for aeronautical services used by civilian aircraft is presented in Fig. 1. This shows that the center frequencies of most of avionic modules are included in a large frequency band between $100 \mathrm{MHz}$ and $12 \mathrm{GHz}$ (X-band). Hence, the RF front-ends used in an SDR need to be agile enough to meet frequency specifications of each system. A challenging task is to meet

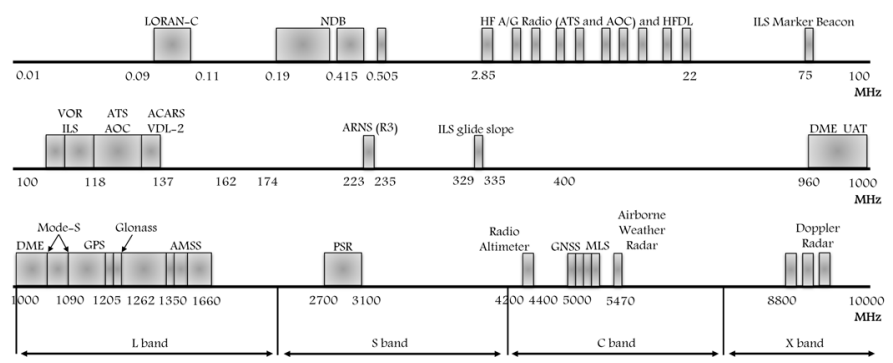

Fig. 1: Allocations spectrum for aeronautical services [1].

the specifications of the local oscillators (LO) which need to cover wide frequency ranges as well as provide high accuracy and good phase noise performance.

Approaches for adding agility to frequency synthesizers have been proposed for several applications in different technologies. Indeed, an agile frequency synthesizer can be provided by using a single voltage controlled oscillator (VCO). The architecture presented in [2] generates a frequency range between $3 \mathrm{GHz}$ and $10 \mathrm{GHz}$ by using four polyphase filters and three single sideband (SSB) mixers. The synthesizer proposed in [3] is able to provide carrier frequencies distributed between $3.4 \mathrm{GHz}$ and $7.9 \mathrm{GHz}$ with two mixers. Moreover, the frequency synthesizer presented in [4] ensures a continuous frequency band between $1.8 \mathrm{GHz}$ and $6 \mathrm{GHz}$ by using wide tuning range $\mathrm{VCO}$ and one SSB mixer. Other proposed systems use a single VCO with switching capacitor bank [5], [6]. This idea allows the extension of the frequency band while keeping low VCO gain. The synthesizer presented in [5] is able to generate the frequency band $9.2 \mathrm{GHz}-12.7 \mathrm{GHz}$ whereas the architecture shown in [6] provides a wide continuous frequency band between $50 \mathrm{MHz}$ and $6 \mathrm{GHz}$.

A wide frequency band can also be provided by employing multiple VCOs. Using this approach, the synthesizer presented in [7] generates a frequency band of $0.4 \mathrm{GHz}-6 \mathrm{GHz}$ by using a dual VCO architecture. The same approach has been used in [8] to generate a frequency band from $1 \mathrm{GHz}$ to $10 \mathrm{GHz}$ spread into thirteen sub-bands. Moreover, twelve bands are provided in [9] between $375 \mathrm{MHz}$ to $6 \mathrm{GHz}$ with a single SSB mixer. For higher frequencies, such as the Ka-band, the designers may use multipliers to reach the desired frequency band. This solution is proposed by [10] with a frequency synthesizer able to generate a frequency band of $21 \mathrm{GHz}-48 \mathrm{GHz}$. 


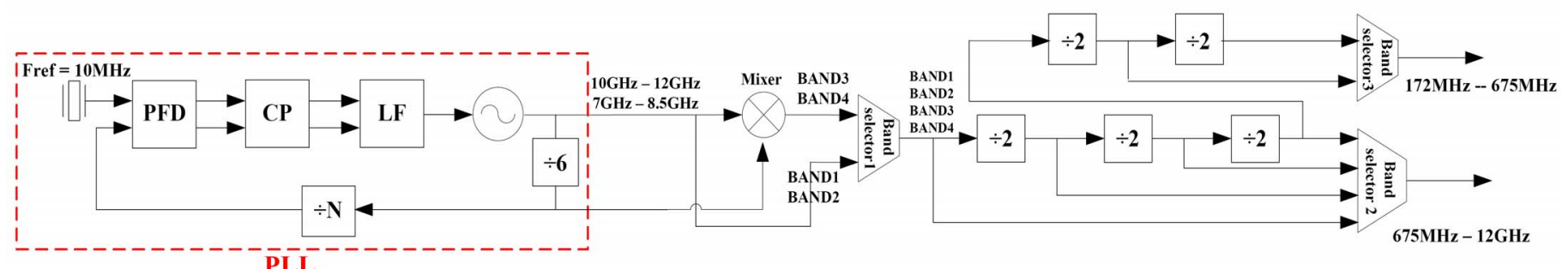

Fig. 2: Block diagram of the frequency synthesizer architecture.

By comparing the previously mentioned approaches, we can see that each architecture exhibits advantages and disadvantages. The evaluation of different synthesizer architectures leads to believe that a frequency synthesizer implemented with one SSB mixer and only a single VCO equipped by a switching inductor and a switching capacitor bank may represent a good trade-off between low power consumption, reduced complexity, and small area, while providing the widest frequency range possible.

Accordingly, the architecture proposed in this paper describes a novel frequency synthesizer architecture for avionic SDR applications that is able to cover a continuous frequency range from $187 \mathrm{MHz}$ to $12 \mathrm{GHz}$. The synthesizer consists of a single wide tuning range VCO controlled with a switching capacitor bank that is included within a phase-locked loop (PLL) and one SSB mixer. Recently, CMOS technology [2], [5] has enabled high degree of integration with good energy efficiency that has enhanced hardware performance. Therefore, $0.13 \mu \mathrm{m}$ CMOS technology will be leveraged in this work.

The rest of the paper is organized as follows. The architecture of the frequency synthesizer is outlined in Section II, followed in Section III by a description of the system components and the core VCO. Simulation results are then presented and commented in Section IV, showing the performance of the proposed architecture, and are followed by the conclusion.

\section{FREQUENCY SYNTHESIZER ARCHITECTURE}

The block diagram of the synthesizer architecture is shown in Fig. 2. It consists of a PLL with a single switched inductor and capacitor bank VCO, a SSB mixer, a charge pump (CP), a phase-frequency detector (PFD), band selectors and frequency dividers.

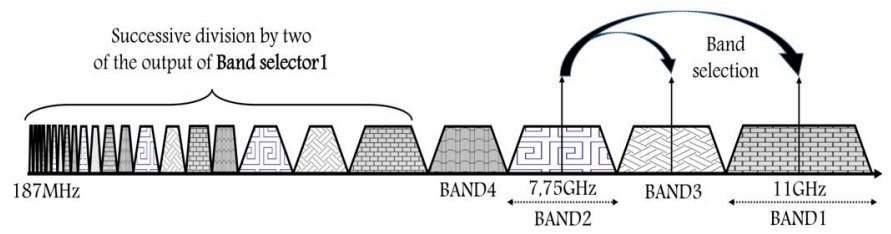

Fig. 3: Conceptual plot of the different frequency allocations.

The VCO provides two frequency bands using the switched inductor: BAND1 from $10 \mathrm{GHz}$ to $12 \mathrm{GHz}$ and BAND2 from $7 \mathrm{GHz}$ to $8.5 \mathrm{GHz}$. The center frequencies are $f_{V C O 1}=11 \mathrm{GHz}$ and $f_{\mathrm{VCO} 2}=7.75 \mathrm{GHz}$ respectively. By dividing BAND1 by six and mixing (through the SSB mixer) the resulting frequency with the BAND1 itself, the mixer output generates BAND3 ranging between $8.5 \mathrm{GHz}$ and $10 \mathrm{GHz}$. The same technique is followed to provide BAND4 from $6 \mathrm{GHz}$ to $7 \mathrm{GHz}$ by mixing the result of BAND2 divided by six with BAND2. Therefore, the band selector (1) can ensure a continuous frequency range from $6 \mathrm{GHz}$ to $12 \mathrm{GHz}$. Furthermore, five frequency bands from $3 \mathrm{GHz}$ to $6 \mathrm{GHz}$, from $1.5 \mathrm{GHz}$ to $3 \mathrm{GHz}$, from $750 \mathrm{MHz}$ to $1.5 \mathrm{GHz}$, from $375 \mathrm{MHz}$ to $750 \mathrm{MHz}$ and from $187 \mathrm{MHz}$ to $375 \mathrm{MHz}$ respectively are obtained by dividing the output of band selector 1 by $2,4,8,16$ and 32 . The architecture frequency allocation is shown in Fig. 3. The considered SSB mixer consists of two 90-degree phase shifters and two double side band mixers as proposed in [11].

\section{ARCHITECTURAL DESIGN}

\section{A. Phase Locked Loop}

The main part of the frequency synthesizer is the PLL presented in Fig. 4. It consists of a frequency reference clock of $f_{\text {ref }}=10 \mathrm{MHz}$, a phase frequency detector with gain of $K_{d}$, a charge pump with current $I_{p}$ where $I_{p}=K_{d} .2 \pi$, a passive low pass filter with a resistor $R$ and two capacitances, $C_{1}$ and $C_{2}$, a VCO with gain of $K_{0}$, a programmable divide-by-N divider, and a divide by six prescaler. In lock, the PLL provides an output frequency $f_{\text {out }}$ such that $f_{\text {out }}=6 . N$. $f_{\text {ref }}=N_{p} . f_{\text {ref }}$.

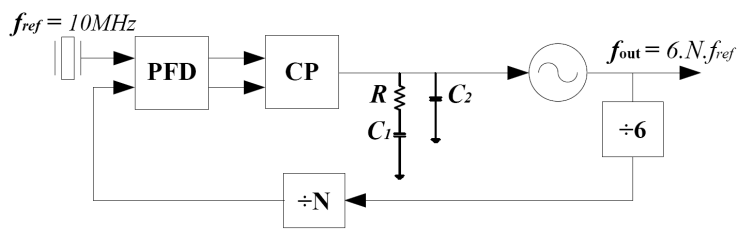

Fig. 4: Phase locked loop block diagram.

The transfer function of the loop filter is given by:

$$
F(s)=\frac{1+R C_{1} s}{s\left(C_{1}+C_{2}\right)\left(1+\frac{R C_{1} C_{2}}{C_{1}+C_{2}} s\right)},
$$

whereas the closed loop transfer function $Y(s)$ can be given by:

$$
Y(s)=\frac{\frac{K_{0}}{s} K_{d} F(s)}{1+\frac{K_{0}}{s} K_{d} F(s) N_{p}}=\frac{\omega_{n}^{2}\left(1+\frac{s}{\omega_{z}}\right)}{s^{2}+2 \xi \omega_{n} s+\omega_{n}^{2}},
$$


where $\omega_{n}$ represents the natural frequency, $N_{p}$ is the division loop factor, $\omega_{z}$ is the stabilizing zero while $\xi$ is the damping factor. Several considerations shall be taken into account when designing the PLL. The wide tuning range requires high VCO gain value. However, the phase noise deteriorates under such condition. For this reason, a trade-off between large VCO tuning range and phase noise performance is always necessary.

Sufficient phase margin is essential for loop stability while achieving high loop bandwidth guarantees short settling time. Therefore, loop filter parameters should be carefully chosen in order to meet the design considerations. For the designed $\mathrm{VCO}$, the $R$ value should be between $5 \mathrm{k} \Omega$ and $20 \mathrm{k} \Omega$ to ensure sufficient phase margin. Capacitance $C_{1}$ is important for loop stability because of its relationship to both the natural frequency and the damping factor. Hence, $C_{1}$ can be between $50 \mathrm{pF}$ and $400 \mathrm{pF}$. Finally, capacitance $C_{2}$ should be less than $10 \%$ of $C_{1}$ in order to preserve the phase margin, and more than $2 \%$ of $C_{1}$ in order to allow for good spur and jitter performance [12]. All design values are selected and optimized through the advanced design system (ADS) simulation tool.

\section{B. Voltage Controlled Oscillator}

Several implementation techniques can be used to design the VCO. For instance, all-PMOS VCOs have better phase noise performance due to the $\mathrm{N}$-wells encompassing the transistors which reduce the impact of substrate noise [13]. However, this approach is limited by a lower tuning range. On the other hand, an all-NMOS VCOs can be more interesting thanks to their larger tuning ranges and smaller footprints compared to that of all-PMOS designs. Therefore, a complementary cross-coupled NMOS and PMOS VCO topology provides a suitable trade-off between tuning range, reduced area and phase noise performance with shorter start-up times than traditional cross-coupled pairs [14]. In addition, the use of a switched capacitor bank and switched inductor improves the phase noise performance by decreasing the VCO gain while maintaining the same covered frequency range.

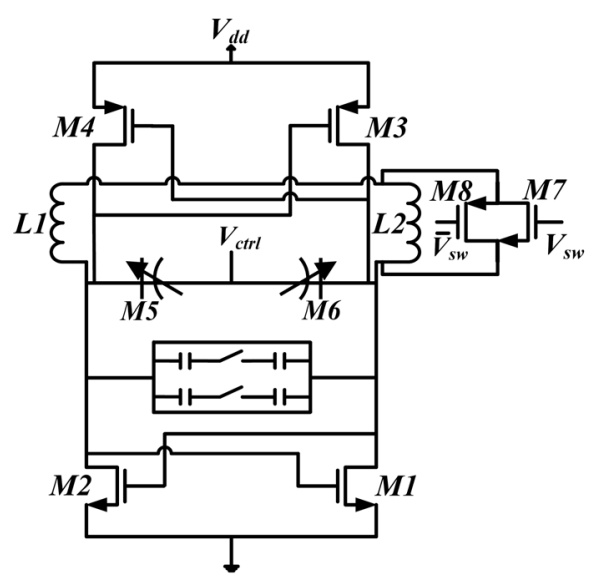

Fig. 5: Schematic of the VCO architecture.

Accordingly, the VCO architecture, presented in Fig. 5, consists of complementary cross-coupled transistors pairs, two NMOS varactors, one transmission gate NMOS/PMOS switch, capacitor bank, and two differentially excited inductors in series, one of which can be shorted. Transistors $M 1-M 4$ form the cross-coupled pairs, while $M 5$ and $M 6$ are used as MOS varactors. $C_{\text {bank }}$ capacitors are controlled by a digital word. Transistors $M 7$ and $M 8$ represent a transmission gate used, as a switch, to short inductor $L 2$. With the switch turned off, the oscillation frequency is given by:

$$
f_{1}=\frac{1}{2 \pi \sqrt{\left(L_{1}+L_{2}\right)\left(C_{\text {var }}+C_{\text {bank }}\right)}},
$$

where $C_{v a r}$ is the tank capacitance, including parasitic and varactors capacitances. Conversely, when $M 7$ is turned on, $L 2$ is shorted, changing the oscillation frequency by offsetting the impact of $L 2$ in (1). Furthermore, changing the $C_{\text {bank }}$ value allows for additional oscillation frequencies. All transistors used as switches should have the smallest possible length in order to ensure fast switching between modes and low ONresistance. The VCO layout is presented in Fig. 6.

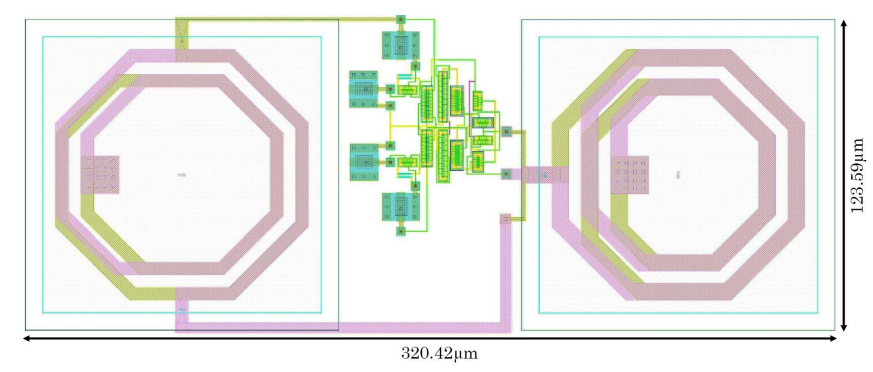

Fig. 6: The VCO layout.

\section{Dividers}

The synthesizer architecture uses several static dividers: a divide-by-2 and a divide-by-3 that are implemented using current mode logic (CML) approach which can reach a frequency operation of up to $40 \mathrm{GHz}$ [15]. The programmable divideby- $N$ in the PLL feedback path is based on the pulse swallow architecture as presented in Fig. 7. This divider consists of a $1 / 2$ dual modulus prescaler, a 7-bit program $P$ counter and a 7-bit swallow $S$ counter such that the achieved divider ratio is given by $P+S$.

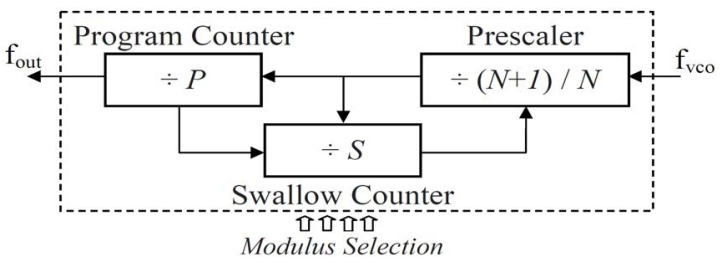

Fig. 7: Block diagram of the programmable pulse-swallow divider.

The prescaler of the pulse-swallow divider is implemented by a divide-by-two that can be selectively bypassed. Thus, this structure is simpler than typical swallow-counter implementations that utilize higher modulus prescalers. To accommodate the full frequency range of the VCO, the programmable divider can be programmed with a ratio from 116 to 200, with $P=115$ and $S$ bounded between 1 and 85, allowing the support of both integer- $N$ and fractional- $N$ implementations. 


\section{Band Selectors}

The band selectors are implemented with transmission gates. Sufficient isolation must be achieved to avoid signal leakage, especially at higher VCO output frequencies [16].

\section{Simulation Results}

The VCO is simulated using the SpectreRF simulator with a $1.2 \mathrm{~V}$ supply. System level simulations are carried out in ADS. The VCO output frequency for each setting of the capacitor bank as a function of the varactor control voltage is shown in Fig. 8. As can be seen, the VCO covers two frequency bands that are enabled by shorting inductor L2 or not. When switch $M 7-M 8$ is ON, BAND1 is provided through overlapping sub-bands ensured by the capacitor bank while BAND2 is provided when $M 7-M 8$ is OFF.

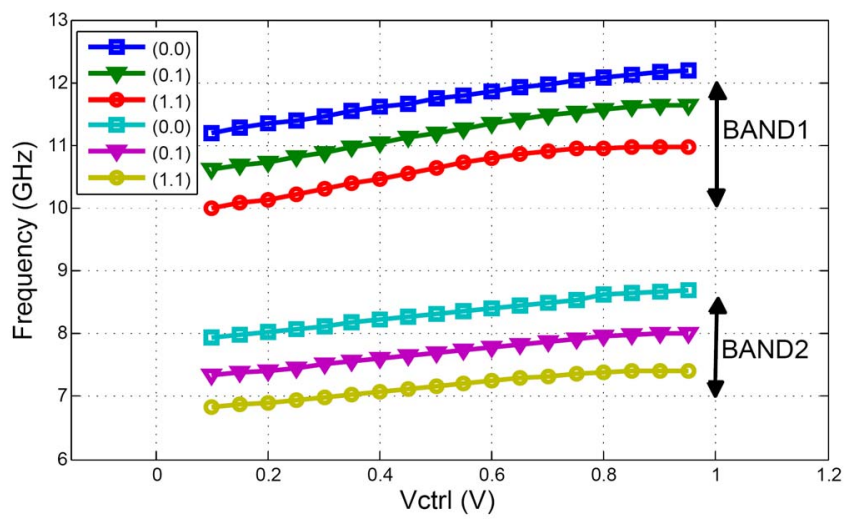

Fig. 8: VCO tuning range for both frequency bands.

The VCO ensures a tuning range of $700 \mathrm{MHz}$ around $11 \mathrm{GHz}$. At $12 \mathrm{GHz}$, the free-running VCO phase noise is of $-125 \mathrm{dBc} / \mathrm{Hz}$ at a $10 \mathrm{MHz}$ offset frequency, whereas the power dissipation is $4.1 \mathrm{~mW}$. The VCO phase noise at a 12 $\mathrm{GHz}$ oscillation frequency is shown in Fig. 9.

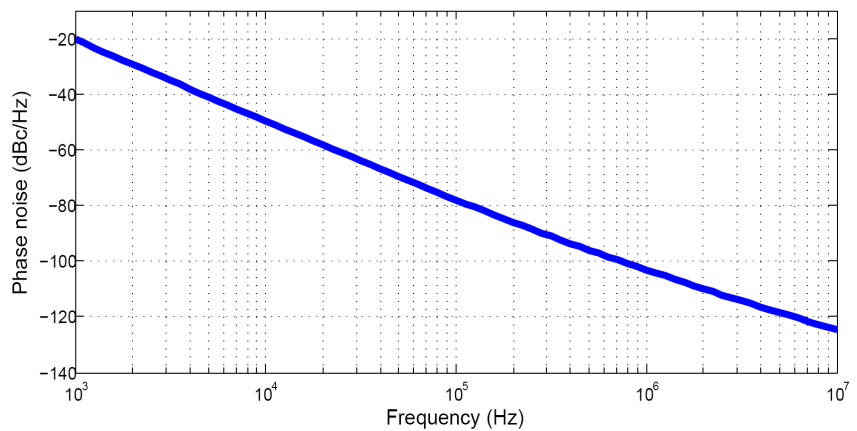

Fig. 9: The VCO phase noise.

The entire synthesizer system is simulated by inserting the VCO gain curves of both frequency bands into the ADS system model. As discussed in Section III, the loop filter components values, VCO gain, $K_{0}$, and charge pump current, $I_{p}$, have to be designed with stability and loop bandwidth trade-offs in mind. With a reference frequency of $10 \mathrm{MHz}$, the values of $R, C_{1}$ and
$C_{2}$ were set to $6 \mathrm{k} \Omega, 356 \mathrm{pF}$ and $30 \mathrm{pF}$, respectively, whereas the charge pump current was fixed to $2 \mathrm{~mA}$. Furthermore, to provide a synthesizer phase noise estimation, the phase noise performance of both the free-running reference, based on results reported in [17], and VCO, from SpectreRF, are inserted within ADS.
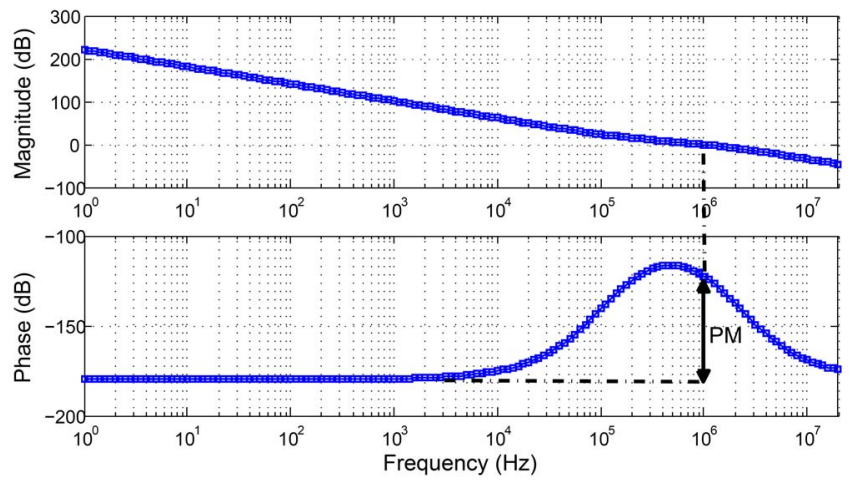

Fig. 10: PLL open-loop frequency response.

The open-loop response of the PLL in BAND1 is obtained by $\mathrm{ADS}$ and presented in Fig. 10. The loop provides a bandwidth of $1.1 \mathrm{MHz}$ and a phase margin of around 58 degrees. Both values allow for a short settling time and stable operation.

The simulated phase noise is shown in Fig. 11 for a $11 \mathrm{GHz}$ output frequency. The $1 \mathrm{MHz}$ offset total phase noise is of $-102 \mathrm{dBc} / \mathrm{Hz}$. The phase noise of the system is dominated by the reference noise at low frequency offsets, which indicates at a higher reference frequency reference could be used to improve close-in phase noise performance. Conversely, the VCO noise becomes the main dominating factor at high frequency offsets, because of the noise sensitivity of the system due to the large VCO gain. As the control voltage of the VCO is limited to $1.2 \mathrm{~V}$, the high VCO gain is necessary in order to ensure a sufficient tuning range. This ultimately limits the system's achievable phase noise performance.

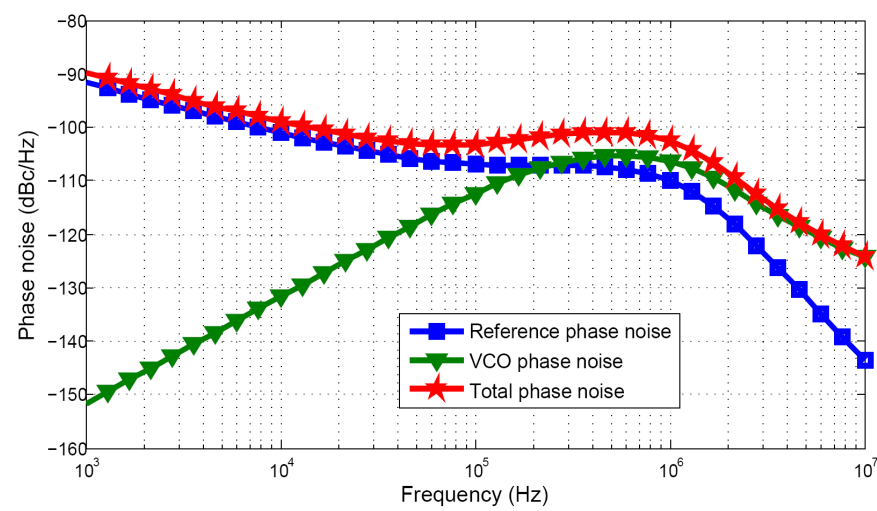

Fig. 11: PLL output phase noise plot for the different noise contributors with the total resulting phase noise.

Typical simulated transient PLL behavior is illustrated in Fig. 12, where the response to the start-up and a $2 \mathrm{GHz}$ frequency jump is shown. After system start-up, the frequency 
TABLE I: Comparison of frequency synthesizers

\begin{tabular}{|c|c|c|c|c|c|}
\hline \hline Characteristics & This work & [5] & [6] & [7] \\
\hline Technology & $0.13 \mu \mathrm{m}$ & $28 \mathrm{~nm}$ & $0.13 \mu \mathrm{m}$ & $0.13 \mu \mathrm{m}$ & 40 \\
Reference frequency (MHz) & 10 & 40 & $18.75-37.5$ & $0.13 \mu \mathrm{m}$ \\
Center frequency (GHz) & $7.75-11$ & 3 & 10 & 25 \\
Output range (GHz) & $0.172 \sim 12$ & $9.2 \sim 12.7$ & $0.05 \sim 6$ & $3.5-6$ \\
Frequency synthesis scheme & Single VCO+Div/Mux & Single VCO+Div & Cap-bank-VCO+Div & Two VCOs+Div/Mux & Dual VCO+Div/Mux \\
Number of mixers & 1 & 0 & 0 & 1 & $0.375 \sim 6$ \\
Loop bandwidth (MHz) & 1.1 & 1.8 & - & 0.1 & 1 \\
Figure-of-merit (FoM) & 225 & 240 & 229 & 0.89 \\
Phase Noise (at 1 MHz) & $-102($ at $11 \mathrm{GHz})$ & $-104($ at $10 \mathrm{GHz})$ & $-110($ at $5.1 \mathrm{GHz})$ & $-114($ at $5 \mathrm{GHz})$ & $-110.8($ at $5.5 \mathrm{GHz})$ \\
\hline
\end{tabular}

shift settling time is of $3.65 \mu$ s for the selected frequency jump.

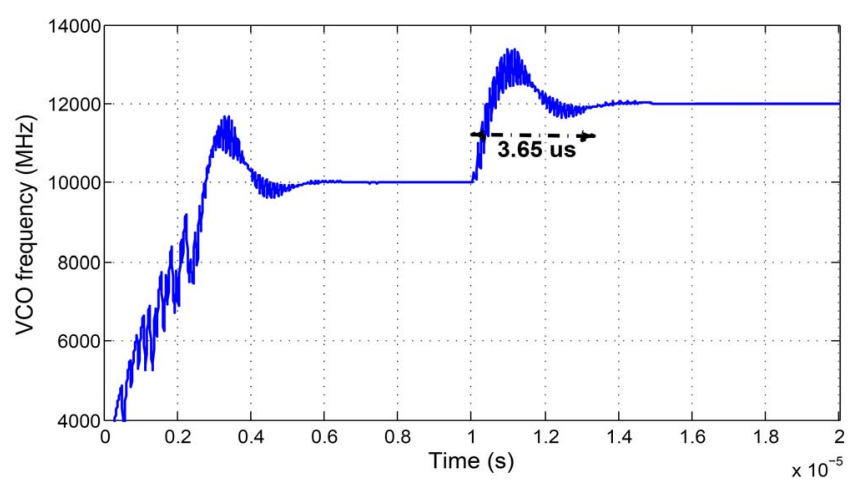

Fig. 12: Typical PLL transient response.

The performance of the proposed architecture is summarised in table I and is compared with other wide frequency range synthesizers previously presented in the literature (for instance, [5], [6], [7] and [9]). The architecture presented in this work provides the widest continuous frequency band with a single SSB mixer and only one VCO, while achieving good phase noise performance.

\section{CONCLUSion}

In this paper, a synthesizer for avionic SDR applications that provides continuous frequencies from $187 \mathrm{MHz}$ to $12 \mathrm{GHz}$ was presented. The proposed frequency synthesizer makes use of one SSB mixer, a single switched capacitor bank and switched inductor VCO. Using ADS-based simulations, the PLL core achieves a loop bandwidth of $1.1 \mathrm{MHz}$ with a phase margin of 58 degrees. At a $11 \mathrm{GHz}$ output frequency, the total phase noise exhibited is $-102 \mathrm{dBc} / \mathrm{Hz}$ at a $1 \mathrm{MHz}$ offset frequency. Ancillary elements of the architecture are currently being implemented in order to send out the chip for fabrication.

\section{REFERENCES}

[1] ICAO, "Aviation Frequency Spectrum and the ITU World Radiocommunication Conferences," march 2015. [Online]. Available: http: //www.icao.int/safety/acp/repository/ITU_presentation_to_C187.pdf

[2] Z.-D. Huang, F.-W. Kuo, W.-C. Wang, and C.-Y. Wu, "A $1.5 \mathrm{~V} 3$ to $10 \mathrm{GHz} 0.18 \mu \mathrm{m}$ CMOS Frequency Synthesizer for MB-OFDM UWB Applications," in Proc. of IEEE MTT-S International Microwave Symposium Digest, June 2008, pp. 229-232.
[3] A. Ismail and A. Abidi, "A 3.1 to $8.2 \mathrm{GHz}$ Zero-IF Receiver and Direct Frequency Synthesizer in $0.18 \mu \mathrm{m}$ SiGe BiCMOS for Mode-2 MBOFDM UWB Communication," IEEE J. Solid-State Circuits, vol. 40, no. 12, pp. 2573-2582, Dec. 2005.

[4] J. Chen, D. Huang, W. Li, J. Zou, and C. Li, "Wideband Fraction-N Frequency Synthesizer Design for Software-Defined Radio," in Proc. of Wireless and Microwave Technology Conference (WAMICON2013), April 2013, pp. 1-6.

[5] K. Raczkowski, N. Markulic, B. Hershberg, and J. Craninckx, "A 9.2-12.7 GHz Wideband Fractional-N Subsampling PLL in $28 \mathrm{~nm}$ CMOS with 280 fs RMS Jitter," IEEE J. Solid-State Circuits, vol. PP, no. 99, pp. 1-11, 2015.

[6] B. Analui, T. Mercer, S. Mandegaran, A. Goel, and H. Hashemi, "A $50 \mathrm{MHz}-6 \mathrm{GHz}, 2-2 \mathrm{MIMO}$, Reconfigurable Architecture, SoftwareDefined Radio in $130 \mathrm{~nm}$ CMOS," in Proc. of IEEE Radio Frequency Integrated Circuits Symposium, June 2014, pp. 329-332.

[7] J. Zhou, W. Li, D. Huang, C. Lian, N. Li, J. Ren, and J. Chen, "A 0.4 to $6 \mathrm{GHz}$ Frequency Synthesizer Using Dual-Mode VCO for SoftwareDefined Radio," IEEE Trans. on Microwave Theory and Techniques, vol. 61, no. 2, pp. 848-859, Feb. 2013.

[8] B. Razavi, "Multi-decade carrier generation for cognitive radios," in Proc. of IEEE Symposium on VLSI Circuits, June 2009, pp. 120-121.

[9] Z. El Alaoui Ismaili, F. Nabki, W. Ajib, and C. Thibeault, "A 0.35-6.25 GHz Cognitive Radio Frequency Synthesizer Architecture," in Proc. of IEEE International Midwest Symposium on Circuits and Systems (MWSCAS), Aug 2013, pp. 796-799.

[10] A. Li, S. Zheng, J. Yin, X. Luo, and H. Luong, "A 21-48 GHz Subharmonic Injection-Locked Fractional-N Frequency Synthesizer for Multiband Point-to-Point Backhaul Communications," IEEE J. SolidState Circuits, vol. 49, no. 8, pp. 1785-1799, Aug 2014.

[11] J. Kim, S. J. Lee, S. Kim, J. O. Ha, Y. S. Eo, and H. Shin, "A 54-862 MHz CMOS Transceiver for TV-band White-Space Device Applications," IEEE Trans. on Microwave Theory and Techniques, vol. 59, no. 4, pp. 966-977, April 2011.

[12] V. F. Kroupa, Phase lock loops and frequency synthesis. John Wiley and Sons,Ltd, June 2003.

[13] R. Aparicio and A. Hajimiri, "A Noise-Shifting Differential Colpitts VCO," IEEE J. Solid-State Circuits, vol. 37, no. 12, pp. 1728-1736, Dec 2002.

[14] C. Shi, H. Yang, H. Xiao, J. Liu, and H. Liao, "A Dual Loop Dual VCO CMOS PLL Using a Novel Coarse Tuning Technique for DTV," in Proc. of IEEE Solid-State ICSICT, Oct 2008, pp. 1597-1600.

[15] Y. Mo, E. Skafidas, R. Evans, and I. Mareels, "A $40 \mathrm{GHz}$ Power Efficient Static CML Frequency Divider in $0.13 \mu \mathrm{m}$ CMOS Technology for High Speed Millimeter-Wave Wireless Systems," in Proc. of Int. Conf. on IEEE Circuits and Systems for Communications, (ICCSC 2008), May 2008, pp. 812-815.

[16] B. Razavi, Phase-Locking in High-Performance Systems: From Devices to Architectures. Wiley-IEEE Press, New York, 2003.

[17] Y. W. Kim and J. D. Yu, "Phase Noise Model of Single Loop Frequency Synthesizer," IEEE Trans. on Broadcasting, vol. 54, no. 1, pp. 112-119, March 2008. 